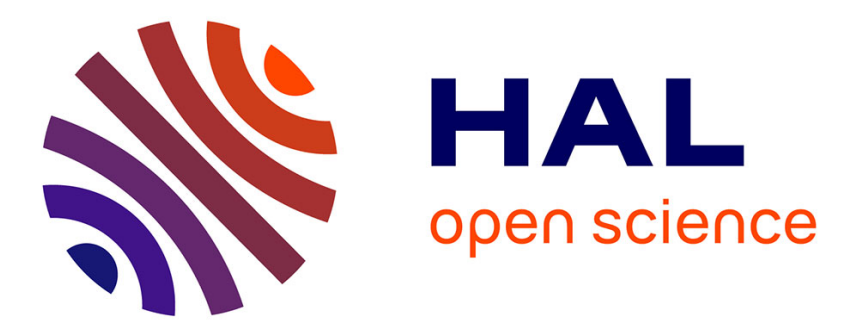

\title{
Observation of 2D Spatiotemporal Rogue Events in a Quadratic Nonlinear Medium
}

Raphael Jauberteau, Alessandro Tonello, Fabio Baronio, Katarzyna Krupa, Guy Mllot, Benjamin Wetzel, Stefan Wabnitz, Vincent Couderc

\section{- To cite this version:}

Raphael Jauberteau, Alessandro Tonello, Fabio Baronio, Katarzyna Krupa, Guy Mllot, et al.. Observation of 2D Spatiotemporal Rogue Events in a Quadratic Nonlinear Medium. CLEO: Applications and Technology, May 2020, Washington, United States. pp.JTu2F.18, 10.1364/CLEO_AT.2020.JTu2F.18. hal-03011031

\section{HAL Id: hal-03011031 https://hal.science/hal-03011031}

Submitted on 18 Nov 2020

HAL is a multi-disciplinary open access archive for the deposit and dissemination of scientific research documents, whether they are published or not. The documents may come from teaching and research institutions in France or abroad, or from public or private research centers.
L'archive ouverte pluridisciplinaire HAL, est destinée au dépôt et à la diffusion de documents scientifiques de niveau recherche, publiés ou non, émanant des établissements d'enseignement et de recherche français ou étrangers, des laboratoires publics ou privés. 


\title{
Observation of 2D Spatiotemporal Rogue Events in a Quadratic Nonlinear Medium
}

\author{
Raphael Jauberteau ${ }^{1,2}$, Alessandro Tonello ${ }^{2}$, Fabio Baronio', Katarzyna Krupa ${ }^{3,4}$, Guy Millot ${ }^{3}$, Benjamin \\ Wetzel $^{2}$, Stefan Wabnitz ${ }^{5,6}$, Vincent Couderc ${ }^{2}$ \\ Dipartimento di Ingegneria dell'Informazione, Università di Brescia, 25123 Brescia, Italy \\ ${ }^{2}$ Université de Limoges, Institut Xlim, UMR CNRS 7252, 87060 Limoges, France \\ ${ }^{3}$ Laboratoire Interdisciplinaire Carnot de Bourgogne, UMR CNRS 6303 - Université Bourgogne Franche-Comté, 21078 Dijon, France \\ ${ }^{4}$ Institute of Physical Chemistry Polish Academy of Sciences, ul. Kasprzaka 44/52, 01-224 Warsaw, Poland \\ ${ }^{5}$ DIET, Sapienza Università di Roma, via Eudossiana 18, 00184 Rome, Italy \\ ${ }^{6}$ Istituto Nazionale di Ottica del Consiglio Nazionale delle Ricerche, Via Campi Flegrei 34, 80078 Pozzuoli (NA), Italy \\ raphael.jauberteau@xlim.fr
}

\begin{abstract}
We experimentally demonstrate 2D quadratic extreme events, appearing and disappearing without a trace. Besides the spontaneous formation of self-guided bicolor solitary waves, ultrafast spatial switching, cascaded events and pulse reshaping are observed. (c) 2020 The Author(s)
\end{abstract}

\section{Introduction}

Solitary waves [1] are widespread in many domains of physics, such as hydrodynamics, plasmas and optics. The soliton concept is also used to describe the observation of extreme events, such as deterministic rogue waves, which appear in nonlinear dispersive or diffractive media because of modulation instabilities. Akhmediev breathers, Peregrine solitons and Kuznetsov-Ma solitons belong to the class of solutions of the nonlinear Schrödinger equation. All of these waves have been interpreted as representing extreme or rogue events, i.e., first appearing and then disappearing, without leaving any significant modification to their local environment. Kerr solitons are well-known from the 70s, whereas quadratic quasi-solitons have been predicted by Karamzin et al. [2] in 1976, and only observed in 1995 by Torruelas et al. [3]. Quadratic quasi-soliton propagation results from modulation instabilities in 2D quadratic materials [4]. Similarly, in 1D wave propagation, the existence of quadratic Peregrine solitons and Akhmediev breathers was predicted by Baronio in 2017 [5].

Here we report, for the first time to our knowledge, the emergence and annihilation of spontaneous 2D quadratic extreme events generated by noncollinear beams with diffraction length much longer than the physical length of the crystal. Spatial switching, multiple rogue wave generation, and temporal reshaping accompany the generation of $2 \mathrm{D}$ extreme waves.

\section{Process}

A Nd:YAG laser delivering 30 ps pulses at $10 \mathrm{~Hz}$ repetition rate was used to inject a large Gaussian beam [radius $\left(1 / \mathrm{e}^{2}\right)=200 \mu \mathrm{m}$ ] at $1064 \mathrm{~nm}$ in a 8 X8X30 mm KTP crystal, cut for type II second harmonic generation. The phase matching process was controlled by the crystal orientation, whereas the input polarization direction and the power could be adjusted by using a polarizer prism placed between two half-wave plates. The spatio-temporal characteristics of the output beam were analyzed with a CCD camera and an autocorrelator, respectively.

Under the influence of crystal birefringence and quadratic nonlinearity, the incident wave $\left(\mathrm{k}_{\omega}\right)$, which is equally split between the ordinary and extraordinary crystal axes, was converted into two noncollinear fundamental frequency $(\mathrm{FF})$ beams, and a third beam at the second harmonic $(\mathrm{SH})$ frequency (see figure 1a).

\section{Results}

At low input power, two FF beams and a weak SH beam propagate in three different directions. By increasing the input peak power, the fundamental beam experiences strong local self-focusing, leading to a stable, 2D localized high-intensity extreme beam (fig. $1 \mathrm{~b}$ ). The radius $\left(1 / \mathrm{e}^{2}\right)$ of this rogue beam is around $30 \mu \mathrm{m}$, i.e., six times smaller than the injected beam. The energy of the $2 \mathrm{D}$ spatial rogue event is close to $3 \%$ of the total FF energy, and its exact amount can be controlled by varying the injected beam width. The peak intensity of the localized extreme beam is about 2.4 higher than the intensity of the output background beam, that emerges unchanged in a linear regime. The spontaneously generated self-guided beam can be obtained for both negative and positive phase mismatch. Under phase matching conditions, the rogue event appears at injected intensities around $0.05 \mathrm{GW} / \mathrm{cm}^{2}$, and it remains stable up to $1 \mathrm{GW} / \mathrm{cm}^{2}$. Above this intensity level, the self-guided beam spreads into several unorganized spots, and eventually it disappears completely, leaving only very small intensity fluctuations on the fundamental beam. The 
pulse temporal profile is also affected by extreme nonlinear propagation, being compressed down to 21 ps, before returning to its original duration (29 ps) as soon as the nonlinear transient event disappears (fig. 1c).
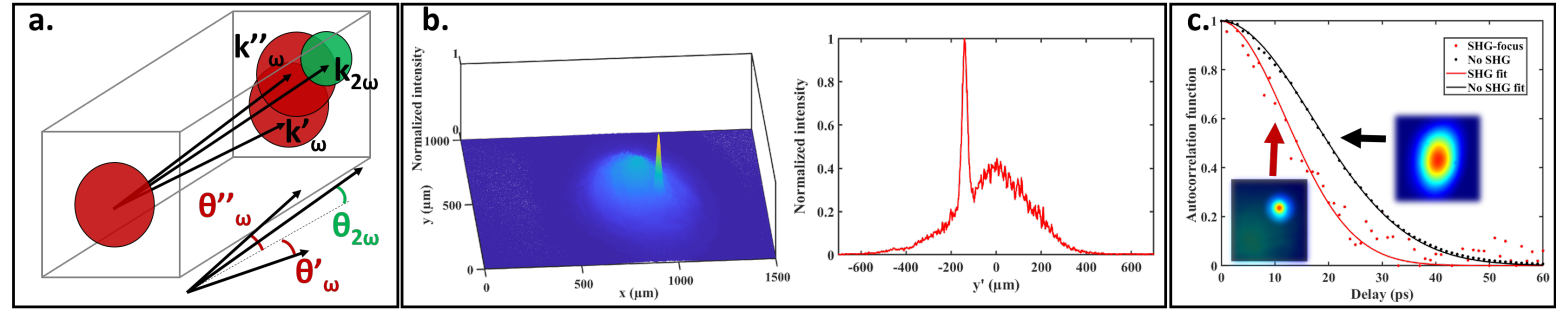

Fig. 1. a. Experimental setup, b. Extreme event at $0.3 \mathrm{GW} / \mathrm{cm}^{2}$ and $\Delta \mathrm{k}=0$, (left) $3 \mathrm{D}$ view, (right) transverse beam profile, c. Autocorrelation profile of the crystal output beam with and without spatial trapping.

In our case, the increasing intensity drives the self-focusing process by means of modulation instability [4]. Because of the 2D nature of the beams, we are far from the experimental conditions leading to 1D Peregrine soliton generation. Since there is a spatial walk-off introduced by the anisotropy of the nonlinear crystal, the self-guided extreme wave generation process undergoes a spatial reorientation, which can be controlled by varying the initial polarization state. As a result, a spatial switching of the rogue wave is observed (fig. $2 \mathrm{a}$ and $2 \mathrm{~b}$ ).

Further increases of the input peak power lead to the generation of additional self-confined events in the transverse output pattern (fig. 2c). Such nonlinear spatial reshaping of the initial beam may also provide a way to control the generation of 2D nonlinear periodic structures, which are reminiscent of the 1D Akhmediev breathers.
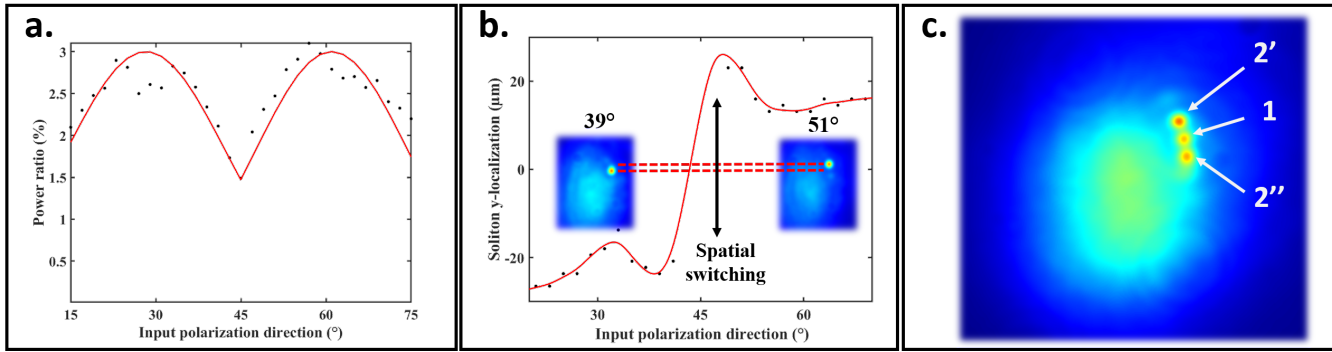

Fig. 2. a. Rogue event power ratio (compared to the whole beam) versus input polarization orientation at 0.66 $\mathrm{GW} / \mathrm{cm}^{2}$ and $\Delta \mathrm{k}=0, \mathbf{b}$. Rogue event position evolution, for a variable input polarization orientation $\left(0.66 \mathrm{GW} / \mathrm{cm}^{2}\right.$ and $\Delta \mathrm{k}=0)$, c. Multiple rogue events generation $\left(0.66 \mathrm{GW} / \mathrm{cm}^{2}\right.$ and $\left.\Delta \mathrm{k}=0\right)$.

\section{Conclusions}

We observed the generation of transient 2D extreme events in a nonlinear quadratic crystal, under experimental conditions far from those leading to quasi-solitons or Peregrine solitons. We observed spatial ultrafast switching and generation of multiple rogue events, which could be exploited for all-optical logic gates. These results can also lead to effective fast saturable absorber functionalities and quadratic beam self-cleaning, as we can force the beam energy to be funneled in a given 1D propagation direction, thus effectively turning a multi-directional beam into a quasiunidirectional beam.

[1] J. Scott Russell, "Report on waves." Fourteenth meeting of the British Association for the Advancement of Science, (John Murray) pp. 311$390(1844)$

[2] Yu. N. Karamzin and A. P. Sukhorukov, "Mutual focusing of high-power light beams in media with quadratic nonlinearity." Sov. Phys. JETP 41, 414 (1976).

[3] W. E. Torruellas, Z. Wang, D. J. Hagan, E. W. Van Stryland, and G. I. Stegeman, "Observation of two-dimensional spatial solitary waves in a quadratic medium." Phys. Rev. Lett. 74, 5036 (1995)

[4] M. Delqué, G. Fanjoux, S. P. Gorza, M. Haelterman, "Spontaneous 2D modulation instability in second harmonic generation process." Opt. Commun. 284, 1401-1404 (2011).

[5] F. Baronio, (2017), “Akhmediev breathers and Peregrine solitary waves in a quadratic medium”. Optics letters, 42(9), 1756-1759. 